CLINICAL STUDY

\title{
Prediabetes is not all about obesity: association between plasma leptin and prediabetes in lean rural Chinese adults
}

Guoying Wang, Xin Liu, Katherine Kaufer Christoffel, Shanchun Zhang, Binyan Wang, Rong Liu, Zhiping Li ${ }^{1}$, Xue Liu ${ }^{1}$, Wendy J Brickman ${ }^{2}$, Donald Zimmerman ${ }^{2}$, Xiping $\mathrm{Xu}^{3}$ and Xiaobin Wang

Mary Ann and J Milburn Smith Child Health Research Program, Department of Pediatrics, Northwestern University Feinberg School of Medicine and Children's Memorial Hospital and Children's Memorial Research Center, Chicago, Illinois 60614, USA, ${ }^{1}$ Institute for Biomedicine, Anhui Medical University, Hefei 230032, People's Republic of China, ${ }^{2}$ Division of Endocrinology, Department of Pediatrics, Northwestern University Feinberg School of Medicine and Children's Memorial Hospital, Chicago, Illinois 60614, USA and ${ }^{3}$ Center for Population Genetics, University of Illinois at Chicago School of Public Health, Chicago, Illinois 60612, USA

(Correspondence should be addressed to X Wang; Email: xbwang@childrensmemorial.org)

\begin{abstract}
Objective: This study investigated the associations of plasma leptin levels with insulin resistance (IR) and prediabetes in relatively lean, rural Chinese men and women.

Design and methods: This study included 574 subjects aged 21-45 years from a community-based twin cohort. Plasma leptin concentrations were measured by sandwich immunoassays using flowmetric xMAP technology. Prediabetes was defined based on fasting plasma glucose and 75-g oral glucose tolerance test. Multivariate linear and logistic regression analyses were used to investigate genderspecific associations of leptin with IR measures and prediabetes, adjusting for intra-twin correlation, measures of adiposity, and other pertinent covariates.

Results: The body mass index is $22.3 \pm 2.7 \mathrm{~kg} / \mathrm{m}^{2}$ in men and $22.5 \pm 2.7 \mathrm{~kg} / \mathrm{m}^{2}$ in women. Leptin levels were positively associated with IR. Individuals with higher tertiles of leptin also had increased risk of prediabetes with odds ratios (OR) of 2.6 (95\% confidence interval (CI): 1.4-5.1) and $4.3(95 \%$ CI: 2.1-8.7) in men; OR of 1.1 (95\% CI: 0.6-2.1) and 3.1 (95\% CI 1.5-6.2) in women for second and third tertile respectively. These associations were attenuated after further adjusting for adiposity measurements only in men. The leptin-prediabetes associations disappeared after adjusting for the homeostatic model assessment of IR in both genders.

Conclusion: In this sample of relatively lean rural Chinese adults, plasma leptin levels were associated with IR and prediabetes in a dose-response fashion, which were not totally explained by adiposity. Our data emphasize that prediabetes is not all about obesity, and leptin may be an additional biomarker for screening individuals at high risk for prediabetes in this population.
\end{abstract}

European Journal of Endocrinology 163 243-249

\section{Introduction}

Prediabetes, characterized by impaired fasting glucose (IFG), impaired glucose tolerance (IGT), or a combined IFG/IGT (1), reflects glucose dysregulation intermediate between normaglycemia and type 2 diabetes mellitus. Prediabetes, often asymptomatic and under-detected, is likely fueling the epidemics of type 2 diabetes and its consequences worldwide (2). Cowie et al. (3) reported that the crude prevalence of prediabetes was almost $30 \%$ in the US adult population. While obesity is a wellrecognized risk factor for prediabetes and type 2 diabetes in heavy western populations, there are paradoxical findings in relatively lean Asian populations. For example, 33\% of US Asian Indian had prediabetes (4). Our own data showed that prevalence of prediabetes is $26 \%$ in middle-aged, rural, relatively lean (mean body mass index (BMI) $21.6 \mathrm{~kg} / \mathrm{m}^{2}$ in men and $22.1 \mathrm{~kg} / \mathrm{m}^{2}$ in women) Chinese population. The high prevalence of prediabetes in relatively lean populations raised a possibility that prediabetes is not all about obesity. A better understanding of the role of other important determinants of prediabetes may provide new insight into the pathogenic mechanisms of prediabetes and type 2 diabetes, and offer a window of opportunity for early detection and prevention.

Leptin, an adipokine encoded by the obese (ob) gene (5), is primarily produced by white adipose cells. It has a variety of important central and peripheral actions, related to the regulation of food intake, energy balance, and metabolism (6,7). Leptin levels in humans increase with increasing body fat, and leptin resistance has been postulated to present in obese individuals. Independent of the relationship to body fat, leptin levels are also positively correlated with fasting insulin levels in humans (8). While the association between leptin and 
insulin has not been fully elucidated, several studies indicated that leptin inhibits insulin synthesis and secretion, while insulin stimulates leptin secretion. This bidirectional hormonal feedback loop between insulin and leptin has been named as the adipoinsular axis (9). To date, few data are available on the role of leptin in prediabetes, especially, in relatively lean Chinese population.

This study was to examine whether leptin is associated with insulin resistance (IR) and prediabetes and whether these associations can be explained by measures of adiposity in relatively lean, rural Chinese adults.

\section{Subjects and methods}

\section{Study population and procedures}

This report utilized data from an ongoing NIH funded study of metabolic syndrome in a large communitybased Chinese twin cohort in Anqing, Anhui province in China during 2005-2006. All the study subjects completed a questionnaire interview, standardized physical examination, dual-energy X-ray absorptiometry (DEXA) scan, and oral glucose tolerance test (OGTT) after at least $10 \mathrm{~h}$ of overnight fasting. We utilized a nested case-control design, which included all prediabetic cases and normal glucose tolerance (NGT) controls matched by age and gender. A total of 574 subjects $(290$ males and 284 females) aged $21-45$ years were included in this analysis. The study protocol was approved by the Institutional Review Boards of Children's memorial Hospital and the Biomedical Institute, Anhui Medical University in Hefei, People's Republic of China. All participants gave written consent.

\section{Physical activity}

Physical activity was assessed using the short version of the international physical activity questionnaire (IPAQShort) (http://www.ipaq.ki.se). Participants were asked information about three types of physical activity (vigorous, moderate, and walking) across various physical activity domains (i.e. leisure time, occupational or school, domestic, and transport), using the 'last 7 days' as the reference period. Using both the total volume of activity and the number of activity days/sessions per week, the IPAQ generates a categorical indicator (low, moderate, and high) of regular physical activity.

\section{Anthropometric parameters}

Height and weight were measured using standard protocols, without shoes and outerwear. Height was measured to the nearest $0.1 \mathrm{~cm}$ on a portable stadiometer and weight to the nearest $0.1 \mathrm{~kg}$. Waist circumference (WC) was measured at the horizontal circumference midway between the lowest rib margin and the iliac crest. BMI was calculated as weight $(\mathrm{kg}) /$ height squared $\left(\mathrm{m}^{2}\right)$. Blood pressure (BP) was measured by trained nurses according to a standard procedure. A standard whole-body scan was performed by DEXA (GE-Lunar Prodigy, Madison, WI, USA) to measure total body fat and trunk fat using a standard software calculation (10). Percentage body fat (PBF) and percentage trunk fat $(\mathrm{PTF})$ were calculated as (total body fat in $\mathrm{kg} /$ weight in $\mathrm{kg}$ ) $\times 100$ and (trunk fat in $\mathrm{kg} /$ total body fat in $\mathrm{kg}) \times 100$ respectively.

\section{Laboratory methods}

Plasma was separated from blood cells in the field within $30 \mathrm{~min}$ after the blood was drawn and kept refrigerated. Plasma glucose was measured within $2 \mathrm{~h}$ by a glucose oxidase method (Hitachi 7020 Automatic Analyzer; Hitachi, Tokyo, Japan). Standard quality control procedures were performed each day with standard samples that came with the reagents (the coefficient of variation, $\mathrm{CV}<8 \%$ ). Triglycerides and cholesterol were measured by enzymatic methods (Boehringer Mannheum, Mannheim, Germany), and high-density lipoprotein cholesterol (HDL-c) was measured by the same enzymatic method after precipitation with dextran sulfate/magnesium chloride. Serum insulin was measured by ECL method on an Elecsys 2010 system (Roche, Basel, Switzerland). Duplicate analyses were also conducted daily using samples collected from study participants $(\mathrm{CV}<10 \%$, mean $=3 \%)$. Plasma leptin concentrations were determined using a sandwich immunoassays based flowmetric xMAP technology on Luminex 200 machines (luminex muti-analyte profiling system, Luminex, Corp., Austin, TX, USA). Immunoassay kit is commercially available from Millipore Corporation (Bedford, MA, USA). Each sample was duplicated, and intra-assay $\mathrm{CV}$ was $<5.1 \%$.

\section{Definition of IR and prediabetes}

All the study subjects completed a standard 75-g glucose OGTT after at least $10 \mathrm{~h}$ of overnight fasting. Blood samples were obtained at 0 and 120 min after glucose administration, for glucose and insulin measurements. IR was estimated using fasting insulin, $2 \mathrm{~h}$ post-load insulin (2 h PI), and the homeostatic model assessment of IR (HOMA-IR). HOMA-IR was calculated using the following formulae: HOMA-IR = fasting insulin concentration $(\mu \mathrm{U} / \mathrm{ml}) \times$ fasting glucose concentration $(\mathrm{mmol} / \mathrm{l}) / 22.5$ (11).

Prediabetes was defined as either IFG (i.e. fasting plasma glucose was between 5.6 and $6.9 \mathrm{mmol} / \mathrm{l}$ ), and/or IGT (i.e. $2 \mathrm{~h}$ post-load glucose was between 7.8 and $11.0 \mathrm{mmol} / \mathrm{l})$.

Exclusion criteria for this analysis included any of the followings: previous diagnosis of diabetes by a physician, plasma glucose concentration $\geq 7.0 \mathrm{mmol} / \mathrm{l}$ after a $10 \mathrm{~h}$ fast, or plasma glucose concentration $\geq 11.1 \mathrm{mmol} / \mathrm{l} 2 \mathrm{~h}$ after a $75-\mathrm{g}$ OGTT, or taking any anti-diabetic medication. 


\section{Statistical analysis}

The distribution of fasting insulin, $2 \mathrm{~h}$ PI, HOMA-IR, and leptin was right skewed, and a logarithmic transformation was used to normalize the data for subsequent statistical analyses. In addition to analyzing leptin as a continuous variable, we also divided leptin into three tertiles, so that we could assess its functional relationship and dose-response associations with IR and prediabetes. We first examined the associations between tertiles of leptin and IR measures using genderspecific multiple linear regression models, with adjustment for age, education (primary school minor or plus), occupation (farmer or nonfarmer), active smoking and passive smoking, alcohol consumption (yes or no), and physical activity (Model A). Since the sample contains 187 twin pairs, we also accounted for intra-twin pair correlation using a generalized estimation equation. We further evaluated whether these associations could be modified by components of metabolic syndrome (Model B) and different adiposity measures (BMI, PBF, PTF, and WC for Model C-F). We also performed similar analyses among two subgroups: subjects with NGT and subjects with prediabetes. To examine explanatory contribution of leptin to IR measures, partial $R^{2}$ was calculated.
Finally, we performed gender-specific multiple logistic regression analyses to assess the associations between leptin tertiles and prediabetes, with the adjustment for the same covariates as listed above plus HOMA-IR (Model G). A two-sided $P$ value $<0.05$ was regarded as statistically significant. We also used Bonferroni correction to address potentially inflated type I error (false positive) due to multiple comparisons (i.e. 4 independent tests), with corrected $P$ value cutoff of 0.0125 . All analyses were performed using SAS version 9.1 (SAS Institute, Cary, NC, USA).

\section{Results}

\section{Demographic, anthropometric, and clinical characteristics}

This study includes 290 males, BMI $22.3 \pm 2.7 \mathrm{~kg} / \mathrm{m}^{2}$ and 284 females, BMI $22.5 \pm 2.7 \mathrm{~kg} / \mathrm{m}^{2}$ and 280 prediabetes cases and 294 NGT controls. Table 1 displays the characteristics of NGT and prediabetes subjects by gender. Smoking status, education level, and occupation are compatible between prediabetes cases and NGT controls. As expected, leptin concentrations,

Table 1 Demographical and clinical characteristics of Chinese men and women aged 21-45 years.

\begin{tabular}{|c|c|c|c|c|}
\hline \multirow[b]{2}{*}{ Variables } & \multicolumn{2}{|c|}{ Men } & \multicolumn{2}{|c|}{ Women } \\
\hline & NGT & PDM & NGT & PDM \\
\hline Age (years) & $36.4 \pm 6.9$ & $34.6 \pm 7.3^{\star \star}$ & $38.1 \pm 5.4$ & $36.3 \pm 5.6^{\dagger}$ \\
\hline Education (\% primary school minor) & 84.4 & 83.2 & 96.6 & 99.3 \\
\hline Occupation (\% farmer) & 76.9 & 67.1 & 70.8 & 70.1 \\
\hline \multicolumn{5}{|l|}{ Smoke status (\% yes) } \\
\hline Active smoking & 67.4 & 59.4 & 3.4 & 4.4 \\
\hline Passive smoking & 70.8 & 73.4 & 76.9 & 70.1 \\
\hline Alcohol consumption (\% yes) & 39.5 & 43.4 & 4.8 & 2.9 \\
\hline \multicolumn{5}{|l|}{ Physical activity } \\
\hline Low & $29(19.7)$ & $17(11.9)$ & $24(16.3)$ & $30(21.9)$ \\
\hline Moderate & $21(14.3)$ & $28(19.6)$ & $27(18.4)$ & 19 (13.9) \\
\hline High & $70(47.6)$ & $73(51.1)$ & $76(51.7)$ & $65(47.5)$ \\
\hline Unknown & $27(18.4)$ & $25(17.5)$ & $20(13.6)$ & $23(16.8)$ \\
\hline PTF (\%) & $53.3 \pm 6.1$ & $55.9 \pm 6.0^{\dagger}$ & $50.7 \pm 4.5$ & $51.4 \pm 4.9$ \\
\hline WC (cm) & $73.2 \pm 7.3$ & $78.1 \pm 9.0^{\dagger}$ & $72.9 \pm 7.2$ & $74.2 \pm 8.6$ \\
\hline $\mathrm{SBP}(\mathrm{mmHg})$ & $108.7 \pm 14.3$ & $113.2 \pm 12.9$ & $108.4 \pm 16.4$ & $107.6 \pm 12.5$ \\
\hline $\mathrm{DBP}(\mathrm{mmHg})$ & $73.4 \pm 11.9$ & $75.8 \pm 10.4$ & $69.9 \pm 12.5$ & $70.4 \pm 9.3$ \\
\hline Triglyceride (mmol/l) & $1.0 \pm 0.6$ & $1.3 \pm 0.8^{\dagger}$ & $1.0 \pm 0.7$ & $1.0 \pm 0.5$ \\
\hline Cholesterol (mmol/l) & $4.1 \pm 0.7$ & $4.4 \pm 0.9$ & $4.1 \pm 0.7$ & $4.2 \pm 0.8$ \\
\hline HDL-c (mmol/l) & $1.7 \pm 0.5$ & $1.8 \pm 0.6$ & $1.6 \pm 0.5$ & $1.7 \pm 0.5$ \\
\hline $\mathrm{FPG}(\mathrm{mmol} / \mathrm{l})$ & $5.1 \pm 0.3$ & $5.8 \pm 0.3^{\dagger}$ & $5.1 \pm 0.3$ & $5.8 \pm 0.4^{\dagger}$ \\
\hline $2 \mathrm{~h}$ PG (mmol/l) & $4.8 \pm 1.3$ & $5.7 \pm 1.7^{\dagger}$ & $5.8 \pm 1.1$ & $6.8 \pm 1.6^{\dagger}$ \\
\hline $\mathrm{FSI}(\mu \mathrm{U} / \mathrm{ml})$ & $4.8 \pm 2.8$ & $7.1 \pm 5.0^{\star}$ & $6.0 \pm 2.6$ & $8.2 \pm 5.2^{*}$ \\
\hline $2 \mathrm{~h} \mathrm{PI}(\mu \mathrm{U} / \mathrm{ml})$ & $21.6 \pm 19.9$ & $30.8 \pm 33.8^{*}$ & $30.1 \pm 16.6$ & $45.9 \pm 36.5^{\star}$ \\
\hline HOMA-IR & $1.1 \pm 0.7$ & $1.8 \pm 1.3^{\star}$ & $1.4 \pm 0.6$ & $2.1 \pm 1.5^{\star}$ \\
\hline Leptin (ng/ml) & $1.9 \pm 1.9$ & $3.4 \pm 3.4^{*}$ & $6.8 \pm 4.0$ & $9.5 \pm 6.8^{*}$ \\
\hline
\end{tabular}

Data are presented as percent and mean \pm S.D.; ${ }^{\star} P<0.05$ for $t$-test based on log-transformed variables; ${ }^{\star \star} P<0.05$ for $t$-test compared with NGT groups; ${ }^{\dagger} P<0.01$ for $t$-test compared with NGT groups; NGT, normal glucose tolerance; PDM, prediabetes (including impaired fasting glucose and impaired glucose tolerance); BMI, body mass index; PBF, percentage body fat; PTF, percentage trunk fat; WC, waist circumference; SBP, systolic blood pressure; DBP, diastolic blood pressure; HDL-c, high-density lipoprotein cholesterol; FPG, fasting plasma glucose; 2 h PG, 2 h post-load glucose; FSI, fasting serum insulin; $2 \mathrm{~h} \mathrm{PI}, 2 \mathrm{~h}$ post-load insulin; HOMA-IR, homeostasis modeling accessible of insulin resistance. 
plasma glucose, insulin measurements, and leptin levels were significantly higher in prediabetes subjects compared with NGT subjects in men and women $(P<0.05)$. Also as expected, adiposity measurements and triglycerides were significantly higher in prediabetes subjects compared with NGT subjects, most notably in men. There were no differences in BP, and plasma HDL-c concentrations between NGT and prediabetes groups. Moreover, the leptin levels were much higher in women than men in both NGT and prediabetes subjects.

\section{Association of plasma leptin with IR}

In both genders, we observed a dose-response relation between the tertiles of leptin and fasting serum insulin (FSI), $2 \mathrm{~h} \mathrm{PI,} \mathrm{and} \mathrm{HOMA-IR} \mathrm{among} \mathrm{all} \mathrm{the} \mathrm{subjects} \mathrm{as}$ well as among the subgroups: subjects with NGT and subjects with prediabetes (Fig. 1). Table 2 showed that higher tertiles of leptin were positively associated with
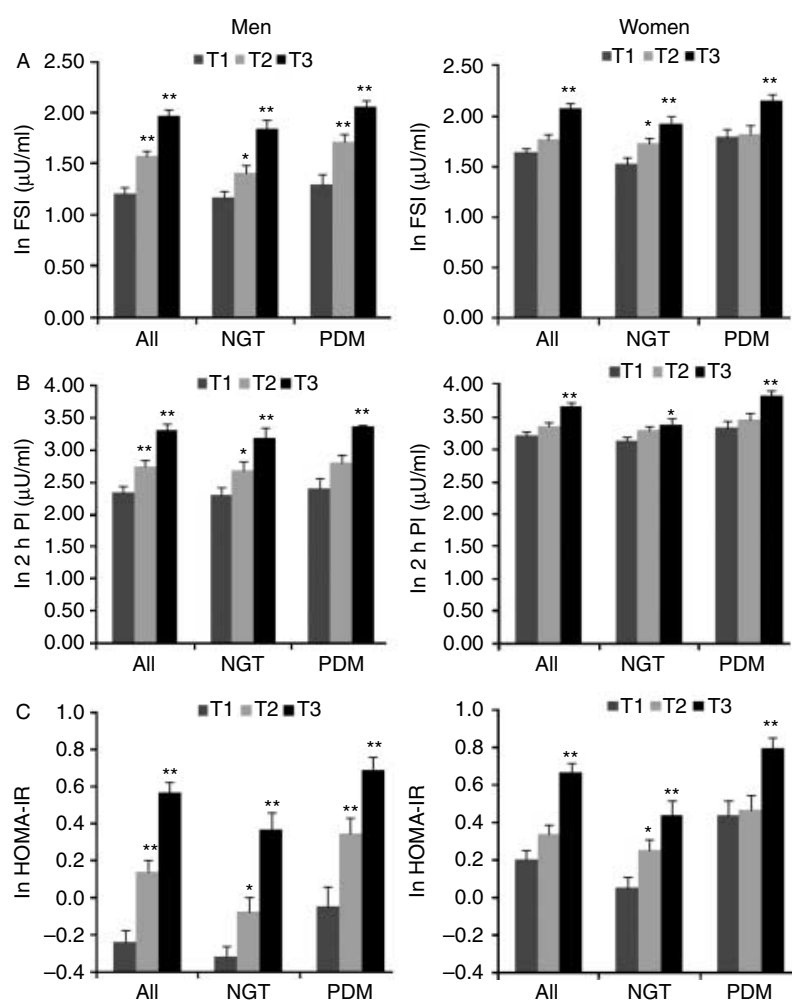

Figure 1 The distribution of insulin resistance measures classified by leptin tertiles, grouped for all subjects (all); subjects with normal fasting glucose (NGT); subjects with prediabetes (PDM). (A) Log-transformed mean fasting serum insulin levels for each tertile of plasma leptin concentrations, FSI, fasting serum insulin. (B) Log-transformed mean $2 \mathrm{~h}$ post-load insulin levels for each tertile of plasma leptin concentrations; $2 \mathrm{~h} \mathrm{PI}$, oral glucose tolerant $2 \mathrm{~h}$ post-load insulin. (C) Log-transformed mean HOMA-IR levels for each tertile of plasma leptin concentrations, HOMA-IR, homeostasis modeling accessible of insulin resistance. ${ }^{*} P<0.05$ compared with first leptin tertile; ${ }^{\star *} P<0.01$ compared with first leptin tertile.
FSI, 2 h PI, and HOMA-IR in both genders. For example, compared with the lowest tertile of leptin, the second and third tertiles of leptin were associated with elevated HOMA-IR with $\beta$ and s.E.M. estimates of $0.32 \pm 0.08$ and $0.71 \pm 0.09$ in men, when adjusting for ages, education, occupation, active smoking and passive smoking, alcohol consumption, and physical activity (Model A). The corresponding estimates for women were $0.15 \pm 0.07$ and $0.50 \pm 0.08$. These associations did not change after adjusting $\mathrm{BP}$ and lipid profile (Model B), while they became attenuated but remained significant after adjusting for adiposity measurements (Model C-F). The explanatory contribution of leptin to HOMA-IR was $21 \%$ in both genders, which was attenuated to 12 and $13 \%$ in men and women respectively after adjusting for $\mathrm{BP}$ and lipid profile. It was further attenuated to $2-10 \%$ after adjustment for adiposity measures. Similar patterns were observed for FSI and $2 \mathrm{~h}$ PI levels.

\section{Association of leptin with prediabetes}

As shown in Table 3, compared with individuals with the lowest tertiles of leptin, those with higher leptin tertiles had increased risk of prediabetes $(\mathrm{OR}=2.6,95 \%$ CI: $1.4-5.1$ and $\mathrm{OR}=4.3$, 95\% CI: $2.1-8.7$ for second and third tertiles in men respectively; $\mathrm{OR}=1.1,95 \% \mathrm{CI}$ : 0.6-2.1 and OR = 3.1, 95\% CI: $1.5-6.2$ for second and third tertiles in women respectively) after adjusting age, education, occupation, smoking and alcohol consumption, and physical activity (Model A). The associations remained after adjusting for BP and lipid profile (Model B). With further adjustment for adiposity measures in the model: BMI (Model C), PBF (Model D), PTF (Model E), and WC (Model F), the associations of leptin with prediabetes were attenuated in men. Adjustment for adiposity measures had no impact on leptin-prediabetes associations in women. Finally, the leptin-prediabetes associations disappeared after adjusting for HOMA-IR (Model G) in both genders.

Of note, we evaluated the associations between leptin and IR measures, leptin and prediabetes stratified by gender, and thus, the significant findings may be false due to the multiple comparisons (i.e. four independent tests). To address this concern, we used Bonferroni correction to address potentially inflated type I error (false positive). Our results showed that the majority of the associations remained statistically significant even after Bonferroni correction, using a cutoff of $P<0.0125$.

\section{Discussion}

This is the first study that assessed gender-specific plasma leptin levels in relation to prediabetes in relatively lean middle-aged rural Chinese subjects. We found significant associations between higher leptin levels and increased risk of prediabetes in both men and 
Table 2 Association between leptin tertiles and insulin resistance measurements in Chinese men and women aged 21-45 years.

\begin{tabular}{|c|c|c|c|c|c|c|}
\hline & \multicolumn{3}{|c|}{ Males } & \multicolumn{3}{|c|}{ Females } \\
\hline & $\begin{array}{c}\text { Second tertile } \\
\beta \text { (S.E.M.) }\end{array}$ & $\begin{array}{c}\text { Third tertile } \\
\beta \text { (S.E.M.) }\end{array}$ & $\begin{array}{c}\text { Inleptin } \\
\text { Partial } R^{2}\end{array}$ & $\begin{array}{c}\text { Second tertile } \\
\beta \text { (S.E.M.) }\end{array}$ & $\begin{array}{l}\text { Third tertile } \\
\beta \text { (S.E.M.) }\end{array}$ & $\begin{array}{c}\text { Inleptin } \\
\text { Partial } R^{2}\end{array}$ \\
\hline \multicolumn{7}{|l|}{ HOMA-IR } \\
\hline Model A & $0.32(0.08)^{\ddagger}$ & $0.71(0.09)^{\ddagger}$ & 0.2084 & $0.15(0.07)^{\star}$ & $0.50(0.08)^{\ddagger}$ & 0.2062 \\
\hline Model B & $0.29(0.08)^{\ddagger}$ & $0.56(0.09)^{\ddagger}$ & 0.1181 & $0.09(0.07)$ & $0.41(0.08)^{\ddagger}$ & 0.1342 \\
\hline Model C & $0.20(0.07)^{\dagger}$ & $0.36(0.09)^{\ddagger}$ & 0.0369 & $0.03(0.07)$ & $0.28(0.09)^{\dagger}$ & 0.0560 \\
\hline Model D & $0.15(0.08)$ & $0.29(0.11)^{\dagger}$ & 0.0201 & $0.03(0.07)$ & $0.26(0.08)^{\ddagger}$ & 0.0532 \\
\hline Model E & $0.29(0.08)^{\ddagger}$ & $0.57(0.09)^{\ddagger}$ & 0.0996 & $0.07(0.07)$ & $0.34(0.08)^{\ddagger}$ & 0.0887 \\
\hline Model F & $0.17(0.07)^{\star}$ & $0.32(0.09)^{\ddagger}$ & 0.0305 & $0.03(0.07)$ & $0.24(0.08)^{\dagger}$ & 0.0392 \\
\hline \multicolumn{7}{|l|}{ FSI } \\
\hline Model A & $0.30(0.07)^{\ddagger}$ & $0.66(0.08)^{\ddagger}$ & 0.1989 & $0.14(0.07)^{\star}$ & $0.47(0.07)^{\ddagger}$ & 0.2062 \\
\hline Model B & $0.27(0.07)^{\ddagger}$ & $0.52(0.08)^{\ddagger}$ & 0.1116 & $0.07(0.06)$ & $0.37(0.07)^{\ddagger}$ & 0.1269 \\
\hline Model C & $0.19(0.07)^{\dagger}$ & $0.34(0.08)^{\ddagger}$ & 0.0342 & $0.02(0.06)$ & $0.25(0.08)^{\ddagger}$ & 0.0510 \\
\hline Model D & $0.14(0.08)$ & $0.26(0.10)^{\dagger}$ & 0.0161 & $0.02(0.06)$ & $0.24(0.08)^{\dagger}$ & 0.0462 \\
\hline Model E & $0.28(0.07)^{\ddagger}$ & $0.53(0.08)^{\ddagger}$ & 0.0961 & $0.06(0.06)$ & $0.31(0.07)^{\ddagger}$ & 0.0828 \\
\hline Model F & $0.17(0.07)^{\star}$ & $0.30(0.09)^{\ddagger}$ & 0.0287 & $0.02(0.06)$ & $0.22(0.07)^{\dagger}$ & 0.0338 \\
\hline \multicolumn{7}{|l|}{$2 \mathrm{~h} \mathrm{Pl}$} \\
\hline Model A & $0.36(0.13)^{\dagger}$ & $0.75(0.14)^{\ddagger}$ & 0.1129 & $0.12(0.07)$ & $0.48(0.09)^{\ddagger}$ & 0.1042 \\
\hline Model B & $0.36(0.14)^{\dagger}$ & $0.68(0.16)^{\ddagger}$ & 0.0908 & $0.08(0.08)$ & $0.40(0.10)^{\ddagger}$ & 0.0664 \\
\hline Model C & $0.30(0.14)^{*}$ & $0.59(0.19)^{\dagger}$ & 0.0549 & $0.10(0.08)$ & $0.43(0.11)^{\ddagger}$ & 0.0595 \\
\hline Model D & $0.10(0.13)$ & $0.11(0.19)$ & 0.0054 & $0.06(0.08)$ & $0.33(0.11)^{\dagger}$ & 0.0219 \\
\hline Model E & $0.33(0.13)^{*}$ & $0.58(0.17)^{\ddagger}$ & 0.0569 & $0.09(0.08)$ & $0.39(0.10)^{\ddagger}$ & 0.0512 \\
\hline Model F & $0.31(0.14)^{*}$ & $0.56(0.19)^{\dagger}$ & 0.0504 & $0.09(0.08)$ & $0.42(0.10)^{\ddagger}$ & 0.0521 \\
\hline
\end{tabular}

${ }^{*} P<0.05 ;{ }^{\dagger} P<0.01$; and ${ }^{\ddagger} P<0.001$ compared with first leptin tertile; HOMA-IR, homeostasis modeling accessible of insulin resistance; FSI, fasting serum insulin; $2 \mathrm{~h} \mathrm{PI}, 2 \mathrm{~h}$ post-load insulin. Model A, adjusted for age, education, occupation, active and passive smoking, alcohol consumption, and physical activity; Model B, Model A plus triglyceride, cholesterol, high-density lipoprotein cholesterol (HDL-C), systolic blood pressure (SBP), diastolic blood pressure (DBP). Model C, Model B plus body mass index (BMI); Model D, Model B plus percentage body fat; Model E, Model B plus percentage trunk fat; Model F, Model B plus waist circumference.

women in a dose-response fashion. The leptin-prediabetes associations were independent of BP and lipid levels. Furthermore, the leptin-prediabetes associations cannot be totally explained by comprehensive measures of adiposity, because further adjusting for adiposity measurements only attenuated these associations in men, and had little effect in women. Our findings reinforce the notion that prediabetes is not all about obesity. If confirmed in future studies, leptin may be an additional biomarker for identifying individuals at high risk of prediabetes in this lean Chinese population.

The biological mechanism by which leptin affects the risk of prediabetes is not completely clear. Notably, the leptin-prediabetes association did disappear after adjusting for HOMA-IR in both genders, suggesting that IR may be a possible biological pathway by which leptin affects prediabetes. The relationship between leptin and insulin appears to be bidirectional, that is, leptin inhibits insulin synthesis and secretion while insulin stimulates leptin secretion (9). The effect of leptin also appears to vary by species (mice versus human) and by clinical conditions. Although leptin improves glucose homeostasis and IR in humans with lipodystrophy or congenital leptin deficiency $(12,13)$, results in humans with 'typical' obesity were disappointing in this regard (14). In the majority of cases of obesity, despite both an intact leptin receptor and high circulating leptin levels, leptin fails to induce weight loss and IR improvement. This diminished response to the anorexigenic and insulin-sensitizing effects of leptin is called 'leptin resistance'. Moreover, attenuation of leptin sensitivity in the brain leads to excess triglyceride accumulation in adipose tissue, as well as in muscle, liver, and pancreas, resulting in impaired insulin sensitivity and secretion (15). Consequently, this could be a sign of decreased inhibition of insulin secretion, and the resulting hyperinsulinemia will further stimulate leptin production. This scenario could lead to what we have observed, that is, high leptin is associated with hyperinsulinemia and prediabetes.

Our finding is consistent with a recent study, in which serum leptin level was higher in those with prediabetes than in the controls in Saudi subjects (16). It is also consistent with previous longitudinal studies on the effects of increased leptin concentrations on diabetes in Caucasian (17) and Japanese American populations (18). In addition, Nowak et al. (19) found that leptin may antagonize some functions of insulin by the attenuation of insulin receptor capacity in liver.

While leptin was independently associated with IR measures and prediabetes in both genders, our data did show some gender differences. First, we found higher leptin levels in females than in males, as reported in previous studies (20). However, this difference was attenuated after adjusting for PBF (data not shown), which is consistent with a previous report that gender difference disappeared when leptin values were compared across similar body fat percentages (21). 


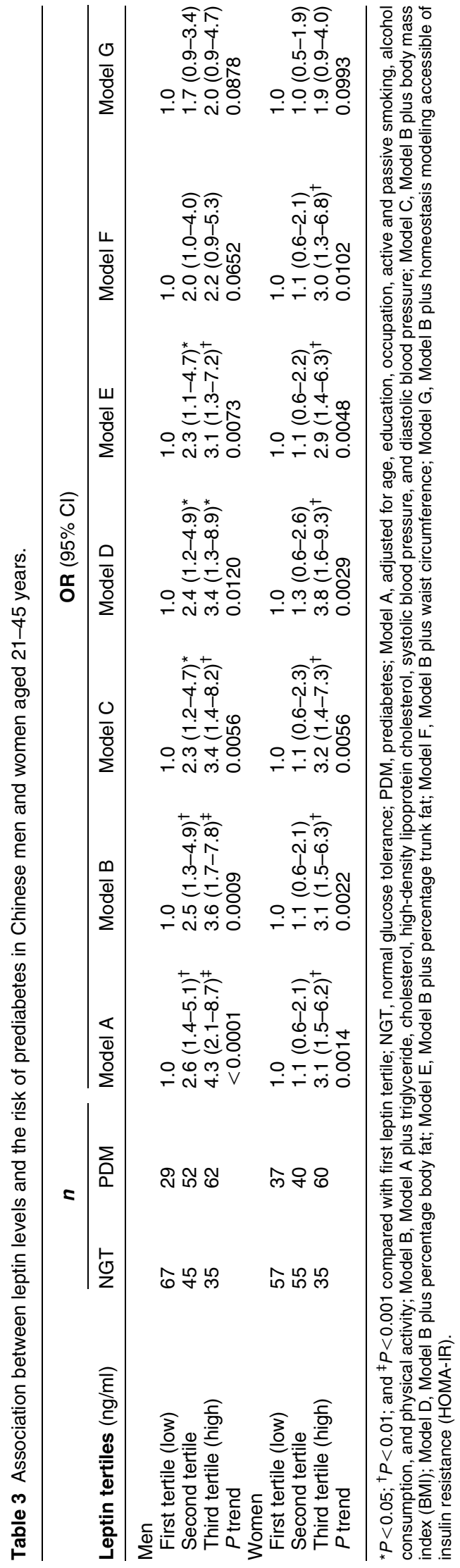

This is consistent with the concept that women have a higher body fat content at a given BMI, and leptin levels are affected by total adiposity. Secondly, our data suggest that adiposity mediated a proportion of the association between leptin and IR measures in both genders and between leptin and prediabetes only in men. One possible explanation for the gender difference is different distributions of fat mass by gender, e.g. more abdominal visceral adipose tissue in men and more subcutaneous adipose tissue mass in women (22). An alternative explanation for the gender difference is that leptin may have differential effects in men and women.

Our study has several strengths. First, we were able to examine a wide array of adiposity measures including BMI, WC, and DEXA-based measurements of body fat, which can more accurately reflect body composition than BMI and WC. Secondly, this study included a relatively homogenous population, in which most of them were farmers, had similar lifestyle and physical activity levels, and dietary intake. The most important feature of our study is that we focused on prediabetes instead of diabetes. This minimizes several sources of confounding variables, such as drug treatment effect, unmeasured metabolic alterations or other subclinical pathology as a result of diabetes, and decreased $\beta$-cell function within diabetic individuals that might obscure secretion and effects of leptin. As such, our study was unlikely to be confounded by the above-mentioned factors. Finally, this population had relatively low plasma insulin levels, which would minimize the confounding of high insulin level on the association between leptin levels and prediabetes.

Our study also has limitations. This cross-sectional analysis only allows for the establishment of an association and not a temporal and causal relationship between leptin and prediabetes. The study sample was nested within a large twin cohort, but we limited to one twin per family for the majority of the sample. For the small number of twin pairs $(n=187)$ included in the analysis, we used general estimating equations to account for intra-pair correlation. Of note, the selected subjects were quite comparable with those excluded from this study with regards to age, gender, and adiposity measures. Furthermore, this communitybased twin population is comparable to local general population as demonstrated in our previous report (23). Although this is among the largest study of this kind in Chinese population, the sample size of our study is modest. We performed a power analysis using QUANTO (http://hydra.usc.edu/gxe) and demonstrated that we have reasonable power $(>85 \%)$ to detect an association with ORs of 2.5 and above (based on the estimates in Table 3 and frequency of exposure to higher leptin levels in normal controls: 0.3-0.4). Lastly, our findings have not been replicated in independent study samples.

In summary, this study demonstrated significant doseresponse associations between plasma leptin level and IR measures and risk of prediabetes in this relatively lean, 
middle-aged rural Chinese population. Leptin appeared to offer additional information beyond adiposity on the risk of prediabetes in this nonobese Chinese sample. Continued follow-up of these subjects will help to evaluate the utility of leptin as a useful predictor of prediabetes and future development of type 2 diabetes.

\section{Declaration of interest}

The authors declare that there is no conflict of interest that could be perceived as prejudicing the impartiality of the research reported.

\section{Funding}

This work was supported in part by the National Institute of Health grant R01 HD049059, R01 HL086461, and R01 AG032227. Dr Xin Liu has been supported in part with Federal funds from the National Institute of Health, through the Clinical and Translational Science Awards Program (CTSA), Northwestern University KL2RR025740.

\section{Acknowledgements}

We would like to thank the study participants and their families, and the faculty and staff of Anhui Medical University.

\section{References}

1 Abdul-Ghani MA, Tripathy D \& DeFronzo RA. Contributions of beta-cell dysfunction and insulin resistance to the pathogenesis of impaired glucose tolerance and impaired fasting glucose. Diabetes Care 200629 1130-1139.

2 Mohamed-Ali V, Pinkney JH \& Coppack SW. Adipose tissue as an endocrine and paracrine organ. International Journal of Obesity and Related Metabolic Disorders 199822 1145-1158.

3 Cowie CC, Rust KF, Ford ES, Eberhardt MS, Byrd-Holt DD, Li C, Williams DE, Gregg EW, Bainbridge KE, Saydah SH \& Geiss LS. Full accounting of diabetes and pre-diabetes in the U.S. population in 1988-1994 and 2005-2006. Diabetes Care 200932 287-294.

4 Misra R, Patel T, Kotha P, Raji A, Ganda O, Banerji M, Shah V, Vijay K, Mudaliar S, Iyer D \& Balasubramanyam A. Prevalence of diabetes, metabolic syndrome, and cardiovascular risk factors in US Asian Indians: results from a national study. Journal of Diabetes and its Complications 200924 145-153.

5 Zhang Y, Proenca R, Maffei M, Barone M, Leopold L \& Friedman JM. Positional cloning of the mouse obese gene and its human homologue. Nature 1994372 425-432.

6 Margetic S, Gazzola C, Pegg GG \& Hill RA. Leptin: a review of its peripheral actions and interactions. International Journal of Obesity and Related Metabolic Disorders 200226 1407-1433.

7 Seufert J. Leptin effects on pancreatic beta-cell gene expression and function. Diabetes 200453 (Suppl 1) S152-S158.

8 Schwartz MW, Prigeon RL, Kahn SE, Nicolson M, Moore J, Morawiecki A, Boyko EJ \& Porte D Jr. Evidence that plasma leptin and insulin levels are associated with body adiposity via different mechanisms. Diabetes Care 199720 1476-1481.

9 Kieffer TJ \& Habener JF. The adipoinsular axis: effects of leptin on pancreatic beta-cells. American Journal of Physiology. Endocrinology and Metabolism 2000278 E1-E14.
10 Pietrobelli A, Formica C, Wang Z \& Heymsfield SB. Dual-energy X-ray absorptiometry body composition model: review of physical concepts. American Journal of Physiology 1996271 E941-E951.

11 Matthews DR, Hosker JP, Rudenski AS, Naylor BA, Treacher DF \& Turner RC. Homeostasis model assessment: insulin resistance and beta-cell function from fasting plasma glucose and insulin concentrations in man. Diabetologia 198528 412-419.

12 Oral EA, Simha V, Ruiz E, Andewelt A, Premkumar A, Snell P, Wagner AJ, DePaoli AM, Reitman ML, Taylor SI, Gorden P \& Garg A. Leptin-replacement therapy for lipodystrophy. New England Journal of Medicine $2002 \mathbf{3 4 6} 570-578$.

13 Farooqi IS, Matarese G, Lord GM, Keogh JM, Lawrence E, Agwu C, Sanna V, Jebb SA, Perna F, Fontana S, Lechler RI, DePaoli AM \& O'Rahilly S. Beneficial effects of leptin on obesity, T cell hyporesponsiveness, and neuroendocrine/metabolic dysfunction of human congenital leptin deficiency. Journal of Clinical Investigation $20021101093-1103$.

14 Hukshorn CJ, Saris WH, Westerterp-Plantenga MS, Farid AR, Smith FJ \& Campfield LA. Weekly subcutaneous pegylated recombinant native human leptin (PEG-OB) administration in obese men. Journal of Clinical Endocrinology and Metabolism 200085 4003-4009.

15 Unger RH. Lipotoxic diseases. Annual Reviews in Medicine 200253 319-336.

16 Montague CT, Farooqi IS, Whitehead JP, Soos MA, Rau H, Wareham NJ, Sewter CP, Digby JE, Mohammed SN, Hurst JA, Cheetham CH, Earley AR, Barnett AH, Prins JB \& O'Rahilly S. Congenital leptin deficiency is associated with severe early-onset obesity in humans. Nature $1997 \mathbf{3 8 7}$ 903-908.

17 Welsh P, Murray HM, Buckley BM, de Craen AJ, Ford I, Jukema JW, Macfarlane PW, Packard CJ, Stott DJ, Westendorp RG, Shepherd J \& Sattar N. Leptin predicts diabetes but not cardiovascular disease: results from a large prospective study in an elderly population. Diabetes Care 200932 308-310.

18 McNeely MJ, Boyko EJ, Weigle DS, Shofer JB, Chessler SD, Leonnetti DL \& Fujimoto WY. Association between baseline plasma leptin levels and subsequent development of diabetes in Japanese Americans. Diabetes Care 199922 65-70.

19 Nowak KW, Mackowiak P, Nogowski L, Szkudelski T \& Malendowicz LK. Acute leptin action on insulin blood level and liver insulin receptor in the rat. Life Sciences 199863 1347-1352.

20 Al-Daghri NM, Al-Attas OS, Al-Rubeaan K, Mohieldin M, Al-Katari M, Jones AF \& Kumar S. Serum leptin and its relation to anthropometric measures of obesity in pre-diabetic Saudis. Cardiovascular Diabetology 2007618.

21 Considine RV, Sinha MK, Heiman ML, Kriauciunas A, Stephens TW, Nyce MR, Ohannesian JP, Marco CC, McKee LJ, Bauer TL \& Caro JF. Serum immunoreactive-leptin concentrations in normal-weight and obese humans. New England Journal of Medicine 1996334 292-295.

22 Demerath EW, Reed D, Choh AC, Soloway L, Lee M, Czerwinski SA, Chumlea WC, Siervogel RM \& Towne B. Rapid postnatal weight gain and visceral adiposity in adulthood: the Fels Longitudinal Study. Obesity 200917 2060-2066.

23 Yu Y, Kumar R, Venners S, Pongracic J, Wang B, Yang J, Li Z, Wang L, Liu X, Tang G, Xing H, Xu X \& Wang X. Age and gender specific lung function predictive equations provide similar predictions for both a twin population and a general population from age 6 through adolescence. Pediatric Pulmonology 2007 42 631-639.

Received 17 April 2010

Accepted 28 May 2010 